\title{
Habilidades sociais educativas: revisão sistemática da produção brasileira
}

\section{Educative Social Skills: A Systematic Review of Brazilian Production Habilidades sociales educatiuas: revisión sistemática de la producción brasilera}

\author{
Joene Vieira-Santos*, Zilda Aparecida Pereira Del Prette, Almir Del Prette \\ Universidade Federal de São Carlos
}

Doi: http://dx.doi.org/10.12804/revistas.urosario.edu.co/apl/a.5069

\section{Resumo}

A expressão "habilidades sociais educativas" (HSE) refere-se aos comportamentos sociais voltados para promover desenvolvimento e aprendizagem do outro. Este estudo visou examinar como a expressão tem sido investigada na literatura brasileira. Buscas nas bases Scielo, Lilacs, Index Psi, Pepsic, Periódicos CAPES, Corpus e Google Acadêmico resultaram em 45 artigos que foram examinados em relação ao ano, autoria, periódico, definição e avaliação de HSE, participantes e região onde ocorreu a coleta. Os dados demonstraram: (i) quatro situações distintas de uso da definição; (ii) quantidade maior de estudos empíricos do que teóricos; (iii) uso predominante de instrumento de relato; (iv) existência de um único instrumento normatizado,apesar da diversidade dos instrumentos identificados; (v) predomínio de estudos sobre a relação pais/cuidadores-filhos; e (vi) concentração das publicações em torno de determinados grupos de pesquisa. Como o mapeamento restringiu-se a artigos publicados em periódicos, sugerem-se pesquisas adicionais com outros tipos de publicações e, possivelmente, explorando análises alternativas.
Palavras-chave: habilidades sociais educativas, revisão de literatura, produção brasileira.

\section{fibstract}

The expression "Educative Social Skills" (ESS) refers to social behaviors to promote development and learning of the other. This study aimed to examine how the expression has been used in Brazilian research literature. Searches on the Scielo, Lilacs, Index Psi, Pepsic, CAPES Journals, Corpus, and Google Scholar data bases recovered 45 papers that were analyzed by publication year, authorship, journal quality, definition and evaluation of ESS, sample, and region of participants. The results showed: (i) four different situations where ESS was used; (ii) more empirical than theoretical studies; (iii) the predominant use of reporting tools for data collection; (iv) just one standardized scale, despite the diversity of instruments identified; (v) the predominance of studies on parent or caregiver-child relationships; and (vi) a concentration of publications around few research groups. As the mapping was restricted to articles published in journals, additional research

* Bolsista CAPES (bacaria CAPES). Correspondência. Correio eletrônico: joenesantos@yahoo.com.br

Cómo citar este artículo: Vieira-Santos, J., Del Prette, Z. A. P., Del Prette, A. (2018). Habilidades sociais educativas: revisão sistemática da produção brasileira. Avances en Psicología Latinoamericana, 36(1), 45-63. doi: http://dx.doi.org/10.12804/revistas. urosario.edu.co/apl/a.5069 
with other types of publications and possibly exploring alternative analyses are suggested.

Keywords: Educative social skills, literature review, Brazilian papers.

\section{Resumen}

La expresión habilidades sociales educativas (HSE) se refiere a los comportamientos sociales dirigidos a promover el desarrollo y aprendizaje de otro. Este estudio buscó examinar cómo la expresión ha sido investigada en la literatura brasileña. Búsquedas en las bases Scielo, Lilacs, Index Psi, Pepsic, Periódicos CAPES, Corpus y Google Académico resultaron en 45 artículos que fueron examinados de acuerdo con el año, autoría, periódico, definición y evaluación de HSE, participantes y región donde se realizó la colecta. Los datos demostraron: (i) cuatro situaciones distintas de uso de la definición; (ii) mayor cantidad de estudios empíricos que teóricos; (iii) uso predominante de instrumento de relato; (iv) existencia de un único instrumento normativizado, a pesar de la diversidad de los instrumentos identificados; (v) predominio de estudios sobre la relación padres/cuidadores-hijos; y (vi) concentración de las publicaciones en torno a determinados grupos de investigación. Como el mapeo se restringió a artículos publicados en periódicos, se sugieren investigaciones adicionales con otros tipos de publicaciones y, posiblemente, explorando análisis alternativos.

Palabras clave: habilidades sociales educativas, revisión de literatura, producción brasilera.

A expressão "habilidades sociais educativas" (HSE) refere-se aos comportamentos sociais voltados para promover desenvolvimento e aprendizagem do outro (Del Prette \& Del Prette, 2010/2001). Em situação formal, Argyle (1967/1994) fazia referência a habilidades sociais do professor no processo de ensino e, no Brasil, na década de 1990, vários estudos focalizaram a avaliação e promoção dessas habilidades no contexto escolar (Del Prette \& Del Prette, 1997; Del Prette, Del Prette, Pontes
\& Torres, 1998; Del Prette, Del Prette, Garcia, Silva \& Puntel, 1998). Possivelmente por isso, as HSE foram inicialmente concebidas como uma das classes de habilidades sociais de trabalho ou profissionais, as quais foram definidas por Del Prette e Del Prette (2010/2001, p.89) como aquelas "que atendem às diferentes demandas interpessoais do ambiente de trabalho, objetivando o cumprimento de metas, a preservação do bem-estar da equipe e o respeito aos diferentes direitos de cada um". Em análises subsequentes, Z. Del Prette e A. Del Prette (2008) detalharam as HSE pertinentes ao desempenho de diferentes agentes, cuja principal função é a tarefa educativa de promover a aprendizagem e o desenvolvimento de outras pessoas, denominando-os de "agentes educativos" e expandindo o uso do termo para todos aqueles que desempenham esse papel social.

De acordo com Manolio (2009), um ponto fundamental da definição de HSE é o caráter "intencional" de tais habilidades, diferindo-as de outras que podem ser desempenhadas por aqueles que assumem o papel de agente educativo. $\mathrm{O}$ uso das HSE implica promover condições para o desenvolvimento e/ou fortalecimento de padrões comportamentais mais efetivos do educando para atuar frente às diversas demandas com as quais se depara. Da forma como os autores definiram, as HSE se aplicam tanto à promoção de comportamentos sociais desejáveis de interação com outras pessoas, como ao processo de ensino e aprendizagem de conteúdos acadêmicos (leitura, escrita, conhecimentos em geral).

No final da década de 1990, os autores começaram a conduzir e orientar pesquisas que utilizavam a noção de habilidades sociais de pais, defendidas como componentes de estratégias, práticas positivas e interações educativas destes com os filhos (Silva, Del Prette \& Del Prette, 2000). Em uma delas, Silva (2000) adotou a expressão $\mathrm{Ha}$ bilidades Sociais Educativas Parentais (HSE-P) para descrever o conjunto das habilidades sociais educativas dos pais na prática educativa positiva 
dos filhos. Segundo Gomide (2003), tais práticas abrangem a monitoria positiva - ou seja, "comportamentos parentais que envolvem atenção para à localização de seus filhos, para suas atividades e formas de adaptação" (p. 24) - e o comportamento moral - ou seja, comportamentos dos pais que auxiliam na transmissão de valores (tais como senso de justiça, empatia, solidariedade, etc.) que impedem o comportamento antissocial.

Com base no questionário de HSE-P, originalmente utilizado na dissertação de mestrado (Silva, 2000) e em desenvolvimentos posteriores desse instrumento, Bolsoni-Silva (2003; 2008; 2009) chega a um sistema de HSE-P composto por três classes amplas: (i) comunicação, referindo-se a comportamentos dos pais de iniciar e manter conversação, perguntar e ouvir com atenção os filhos; (ii) expressar sentimentos e enfrentamento, envolvendo expressar sentimentos positivos e negativos, expressar opiniões, demonstrar carinho e brincar com o filho; e (iii) estabelecimento de limites, o que inclui identificar os motivos pelos quais se estabelece limite, descrever os comportamentos considerados apropriados ou inapropriados aos filhos, cumprir promessas, conversar com o cônjuge para estabelecer concordância nas práticas educativas, reconhecer os próprios "erros" e identificar ocasiões e comportamentos que justificam o estabelecimento de limites. Investindo na descrição contextual do desempenho dessas classes, propõe a análise das condições antecedentes e/ou consequentes às respostas dos pais/cuidadores, em termos dos comportamentos do(s) filho(s) que afetam o desempenho das HSE-P. Essas categorias subsidiaram a elaboração do Roteiro de Entrevista de Habilidades Sociais Educativas Parentais (REHSE-P, Bolsoni-Silva, 2008) e sua validação psicométrica subsequente (Bolsoni-Silva \& Loureiro, 2010) com resultados satisfatórios de consistência interna e validade, e uma estrutura fatorial composta por dois fatores: características positivas do relacionamento pais e filhos e características negativas do relacionamento pais e filhos.
A partir de pesquisas empíricas e elaboração conceitual, Z. Del Prette e A. Del Prette (2008) propuseram um sistema amplo de HSE composto por quatro grandes classes: (i) estabelecer contextos interativos potencialmente educativos, referindo-se a comportamentos que promovem a organização do material, do contex to físico ou social para proporcionar a interação educativa; (ii) transmitir ou expor conteúdos sobre habilidades sociais, ou seja, comportamentos que apresentam conteúdos de conhecimento sobre habilidades sociais, podendo ou não ser mediados por recursos audiovisuais; (iii) estabelecer limites e disciplina, isto é, comportamentos que estabelecem regras, normas ou valores; e (iv) monitorar positivamente, ou seja, administrar contingências sobre o comportamento diretamente observável ou sobre o comportamento relatado pelo educando, promovendo consequências reforçadoras para os desempenhos sociais desejáveis observados ou relatados. Tal sistema, segundo os autores, tem se mostrado adequado para examinar as interações entre agentes educativos e educandos em situação natural e estruturada, não se restringindo apenas ao âmbito escolar, mas estendendo-se a qualquer contexto educativo, como o que ocorre, por exemplo, em empresas e outros contextos sociais como sindicatos.

A partir do que foi exposto, o presente trabalho teve como objetivos: (i) mapear a produção acadêmica brasileira que tem enfocado as HSE, e (ii) identificar os tipos de estudos que têm sido desenvolvidos. Isto parece ser relevante por alguns motivos. Primeiramente, para examinar a abrangência do uso da expressão HSE em termos de públicos-alvo junto aos quais tais habilidades têm sido investigadas. Em segundo lugar, para verificar como as pesquisas têm se distribuído em termos de localização geográfica - ou seja, regiões do país em que tais estudos estão sendo realizados -, avaliando se este é um termo amplamente difundido entre pesquisadores ou se tem se restringido a determinados grupos de pesquisa. Em terceiro lugar, também pode ser útil para verificar quais 
instrumentos têm sido utilizados para avaliar o repertório de HSE e a forma como os mesmos são empregados. Por fim, examinar como as HSE têm sido investigadas na literatura parece importante no sentido contribuir para elucidar questões pertinentes às semelhanças e divergências entre os sistemas descritos acima.

\section{Método}

\section{Coleta dos dados}

O presente estudo consistiu em uma revisão de literatura, sobre o estado da arte em HSE (Von Hoendorff, 2014). As buscas foram realizadas em novembro de 2015, empregando-se a expressão "habilidades sociais educativas" nas seguintes bases de dados: Scielo Brasil, Lilacs, Index Psi, Pepsic, Periódicos CAPES, Corpus e Google Acadêmico. Em um primeiro momento, foram selecionados para análise os artigos que possuíam tal expressão no título, resumo, palavras-chave e/ou no texto.

Para textos cujo conteúdo completo não estava disponibilizado na base de dados que os citava, foram realizadas buscas em outros sites (tais como Google) para localizar o texto na íntegra. Caso não fosse possível localizar o texto completo, o mesmo era automaticamente excluído da amostra de artigos a serem analisados posteriormente. Além disso, quando a base de dados trazia como resultado da busca, trabalhos em inglês ou espanhol, para identificar se o mesmo deveria ou não ser selecionado para análise, usou-se termos equivalentes à expressão: "social educative skills" e "habilidades sociales educativas", respectivamente.

No Google Acadêmico, para restringir o número de resultados apresentados, a busca foi feita colocando-se a expressão entre aspas e excluindo os resultados classificados pelo site como patentes ou citações. Ainda com estas precauções, foram identificados 5360 trabalhos (536 páginas com 10 resultados em cada uma). Dado o grande número de resultados trazidos por esta ferramenta de busca, a análise dos mesmos foi interrompida no momento em que se passaram duas páginas consecutivas sem a localização de artigos novos - ou seja, não identificados através das buscas nas demais bases de dados. Foram analisadas as 14 primeiras páginas apresentadas na busca realizada em 16/11/2015.

Por fim, além destas bases de dados, também foi consultada a seção de "artigos publicados em periódicos" da página do Grupo de Relações Interpessoais e Habilidades Sociais (RIHS) da Universidade Federal de São Carlos (UFSCar): www.rihs. usfcar.br. Essa página foi incluída entre as bases de dados empregadas, visto que os coordenadores deste grupo de pesquisa foram os primeiros autores a usar/popularizar a expressão HSE.

\section{Análise dos dados}

A exclusão dos artigos duplicados e daqueles que não tratavam do tema de interesse desta pesquisa resultou no número inicial de artigos rastreados. Destes, foram excluídos, em seguida, os artigos que apenas citavam a expressão HSE na seção de referências. Os artigos classificados como elegíveis para compor o corpus desta pesquisa foram então lidos na íntegra e divididos em duas categorias: (i) artigos que apenas citavam as HSE e (ii) artigos nos quais as HSE eram o foco do estudo. O primeiro grupo foi excluído e, para as análises conduzidas neste trabalho, foram considerados apenas os artigos nos quais as HSE eram o foco da investigação. Estes foram examinados a partir dos seguintes critérios: autoria, periódicos no qual os artigos foram publicados, definição dada à expressão HSE, tipo de estudo, como as HSE eram avaliadas, instrumentos utilizados, participantes e região do país no qual os dados foram coletados.

\section{Resultados e Discussão}


A Figura 1 apresenta o fluxograma da coleta e análise de dados. Como é possível observar, dos 90 artigos rastreados, 16 foram excluídos da amostra porque apenas citavam a expressão HSE na seção de referências, sem mencioná-la ao longo do texto. Dos 74 artigos elegíveis, 29 foram excluídos porque as HSE eram citadas, mas não eram o foco do estudo. Desta forma, o corpus desta pesquisa foi composto por 45 artigos que possuíam as HSE como foco do estudo e cujo texto completo encontrava-se disponível, os quais foram examinados a partir das categorias de análise adotadas.

A distribuição temporal dos artigos demonstrou que os trabalhos mais antigos identificados foram publicados no ano de 2002, o que dá a entender que publicações em periódicos científicos sobre HSE surgem logo após a expressão ser apresentada na taxonomia das habilidades sociais proposta por Del Prette e Del Prette (2010/2001). Também foi possível perceber uma oscilação na quantidade de publicações sobre o tema ao longo do tempo, sendo que 2010 e 2008 foram os anos nos quais foi publicada a maior quantidade de manuscritos, respectivamente, nove e sete artigos. Nos demais anos a quantidade de artigos variou entre um e quatro.

Em relação à autoria, dois tipos de dados foram extraídos: a quantidade de artigos que cada autor publicou e a quantidade de autores em cada artigo. Foram identificados 57 autores distintos, sendo que 38 deles escreveram um único artigo e 11 deles escreveram dois artigos cada. Quatro autores se destacam quanto ao número de artigos que publicaram na área, a saber: Bolsoni-Silva, Z . Del Prette, Cia eA. Del Prette, possuindo, respectivamente, 22, 11, 9 e 9 publicações cada um, o que são indícios que estes são os autores que mais tem pesquisado sobre o tema das HSE. Já com respeito à quantidade de autores por artigos, verificou-se uma variação entre dois e seis autores, sendo que

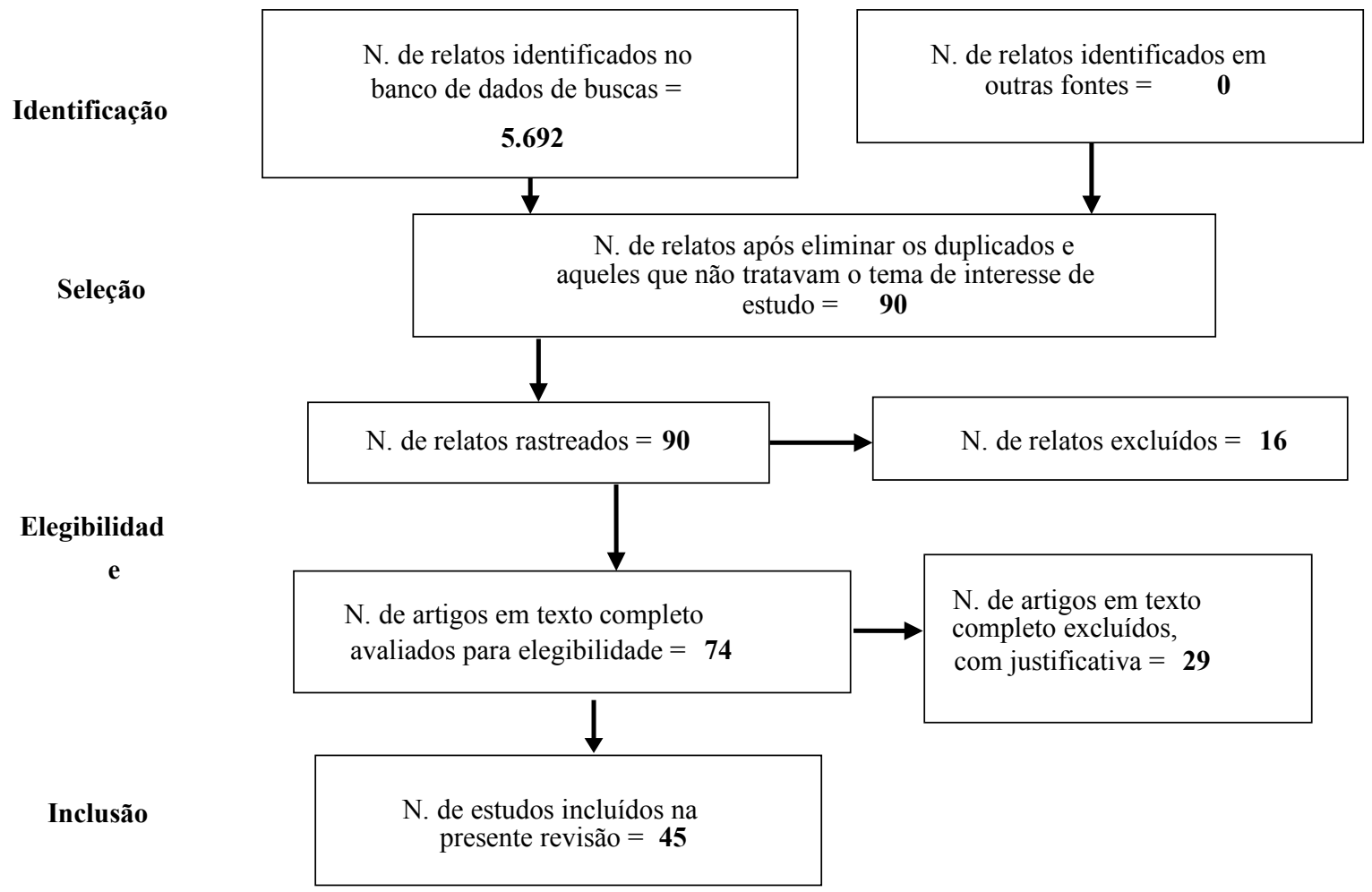

Figura 1. Fluxograma da seleção e análise de artigos para a presente revisão (seguindo proposta de Galvão, Pensani \& Harrad, 2015) 
$80,0 \%$ das publicações analisadas haviam sido escritas por dois ou três autores.

Os periódicos científicos nos quais estes artigos foram publicados eram destinados a estudos da área da Psicologia ou da Educação, o que parece ser coerente com o campo de conhecimento no qual a HSE surgiu e tem se desenvolvido. Os artigos foram publicados em 24 periódicos científicos diferentes, sendo que aqueles que possuíam maior número de artigos sobre o tema foram Paidéia (Ribeirão Preto), Aletheia, Estudos e Pesquisas em Psicologia e Psico (Porto Alegre) com, respectivamente, 4, 4, 3 e 3 artigos publicados.

Quanto ao sentido da expressão HSE, verificou-se que: (i) 15 estudos empregam a expressão no sentido mais amplo, conforme proposto por Del Prette e Del Prette (2010/2001); (ii) 20 estudos utilizam a expressão HSE-P, conforme sugerido por Silva (2000); (iii) quatro estudos usam a expressão como sinônimo de práticas educativas positivas, estas entendidas como uma combinação envolvendo HSE e variáveis de contexto; e (iv) seis estudos apenas citam a expressão, sem qualquer definição para a mesma. Entre os estudos que usaram a expressão no sentido mais amplo, 12 referiam-se a relação pais/cuidadores-filhos, dois a relação professor-aluno e um apresentava um sistema de classificação das HSE, sem restringi-lo a um tipo específico de agente educativo. Todos os estudos que empregaram a expressão HSE-P referiam-se a relação pais/cuidadores-filhos. Nos estudos que usavam a expressão como sinônimo de práticas educativas positivas, o significado atribuído à mesma parece estar relacionado à expressão HSE-P, tal como proposto por Silva (2000), visto que em tais estudos o instrumento utilizado para coleta de dados era o Roteiro de Entrevista de Habilidades Sociais Educativas Parentais (RE-HSE-P) ou o Questionário de Habilidades Sociais Educativas para Professores (elaborado a partir do RE-HSE-P). Além disso, neste grupo de estudos, um deles estava relacionado à relação pais-filho e os outros três à relação professor-aluno.
Na tabela 1 encontram-se os dados referentes ao tipo de estudo, como as HSE foram avaliadas, participantes e região na qual os dados foram coletados. É possível observar que cinco artigos referiam-se a trabalhos teóricos e 40 a pesquisas empíricas. Os estudos teóricos foram divididos em ensaio, revisão de literatura e construção de sistema, sendo que entre os quatro artigos que compunham as categorias ensaio e revisão de literatura, três deles abordavam questões relativas às HSE e inclusão (Rosin-Pinola \& Del Prette, 2014; Rocha, Andrade \& Dourado, 2011; Rocha $\&$ Del Prette, 2010). É possível que isto esteja relacionado à ampliação do campo teórico-prático das habilidades sociais por meio de dissertações e teses que foram depois publicadas sob a forma de artigos e que foram estudos produzidos no âmbito da Educação Especial e da importância dos estudos sobre inclusão nessa área (A. Del Prette \& Z. Del Prette, 2008).

Ainda em relação à proporção entre artigos teóricos e artigos empíricos, a grande quantidade de estudos empíricos $(88,9 \%)$ parece ser um dado positivo, pois pode indicar que as formulações conceituais iniciais foram heurísticas na indução de pesquisas nessa área e que o conhecimento relativo às HSE está se estabelecendo a partir de dados empíricos, o que tende a sugerir maior robustez na elaboração do mesmo.

Os estudos empíricos foram divididos em pesquisa sem intervenção (30 artigos) e pesquisa com intervenção (10 artigos). Além disso, os estudos empíricos também foram analisados quanto aos participantes, à maneira como as HSE foram avaliadas, à região na qual os dados foram coletados e ao instrumento de autorrelato utilizado para mensurar as HSE.

A maioria dos estudos empíricos foi realizada com cuidadores primários, sendo possível encontrar pesquisas que envolviam a participação de ambos os pais (casal), apenas da mãe, apenas do pai e de outros cuidadores (tia e avó). Apenas $12,5 \%$ dos estudos foram realizados junto a 
Tabela 1

Dados relacionados ao tipo de estudo, objetivos dos estudos, participantes, como as HSE foram avaliadas e região na qual os dados foram coletados

\begin{tabular}{|c|c|c|c|}
\hline \multicolumn{2}{|r|}{ Categorias de análise } & Frequência & $\%$ \\
\hline \multicolumn{4}{|c|}{ Tipo de estudo } \\
\hline \multirow{3}{*}{ Teórico } & Ensaio & 3 & 6,7 \\
\hline & Revisão de literatura & 1 & 2,2 \\
\hline & Elaboração de sistema & 1 & 2,2 \\
\hline \multirow{2}{*}{ Empírico } & Pesquisa sem intervenção & 30 & 66,7 \\
\hline & Pesquisa com Intervenção & 10 & 22,2 \\
\hline Total & & 45 & 100,0 \\
\hline \multicolumn{4}{|c|}{ Participantes* } \\
\hline \multicolumn{2}{|c|}{ Casal (pai e mãe) } & 5 & 12,5 \\
\hline \multicolumn{2}{|c|}{$\begin{array}{l}\text { Cuidadores primários (pai, mãe, avó } \\
\text { ou tia) }\end{array}$} & 12 & 30,0 \\
\hline \multicolumn{2}{|l|}{ Mães } & 10 & 25,0 \\
\hline \multicolumn{2}{|c|}{ Pais (homens) } & 4 & 10,0 \\
\hline \multicolumn{2}{|c|}{ Pais (pai ou mãe) e crianças } & 1 & 2,5 \\
\hline \multicolumn{2}{|c|}{ Mães e adolescentes } & 1 & 2,5 \\
\hline \multicolumn{2}{|c|}{ Professores } & 4 & 10,0 \\
\hline \multicolumn{2}{|c|}{ Professores e alunos } & 1 & 2,5 \\
\hline \multicolumn{2}{|l|}{ Crianças } & 2 & 5,0 \\
\hline \multicolumn{2}{|l|}{ Total } & 40 & 100,0 \\
\hline
\end{tabular}

Maneira como as HSE foram avaliadas*

\begin{tabular}{lcr} 
Autorrelato & 27 & 67,5 \\
Autorrelato + Relato de terceiros & 3 & 7,5 \\
Autorrelato + Observação & 3 & 7,5 \\
Relato de terceiros & 2 & 5,0 \\
Não avaliadas diretamente & 4 & 10,0 \\
Não foi descrita & 1 & 2,5 \\
Total & 40 & 100,0 \\
\hline \multicolumn{1}{c}{ Região em que os dados foram coletados* } \\
Centro-Oeste & 1 & 2,5 \\
Sudeste & 31 & 77,5 \\
Sul & 4 & 10,0 \\
Não especificada & 4 & 10,0 \\
Total & 40 & 100,0 \\
\hline
\end{tabular}

Nota. * Dados relativos apenas aos estudos empíricos. professores, sendo que destes apenas um estudo referia-se a intervenção com este público específico (Fornazari et al., 2014). A função de agente educativo, segundo Z. Del Prette e A. Del Prette (2008), é desempenhada por qualquer indivíduo que assume a tarefa educativa, ou seja, a tarefa de promover a aprendizagem e desenvolvimento de outro. Falando especificamente sobre a promoção de habilidades sociais, estas são desenvolvidas inicialmente a partir das relações estabelecidas entre criança e cuidadores; contudo, à medida que ela passa a inserir-se em novos contextos sociais, tal como a escola, outras pessoas passam a exercer a função de agente educativo na vida da mesma, como por exemplo, professores. Durante a formação acadêmica/escolar, os professores influenciam de maneira relevante as diversas dimensões relacionadas ao desenvolvimento de uma criança, entre eles, a dimensão social. Assim, os dados do presente estudo parecem indicar a necessidade de mais pesquisas que enfoquem questões relativas às HSE de professores, dos diferentes níveis de ensino. Nessa direção, podem ser referidos os estudos em andamento no Grupo "Relações Interpessoais e Habilidades Sociais" (www.rihs.ufscar. br), voltados para a construção do Inventário de Habilidades Sociais Educativas de Professores da Educação Básica (IHSE-Prof, Del Prette \& Del Prette, 2013b) e de Professores Universitários (IHSE-PU, Del Prette \& Del Prette, 2013c), que podem viabilizar a ampliação de pesquisas futuras na direção de suprir esse descompasso.

Também é possível observar na tabela 1 que, em $80,0 \%$ dos estudos empíricos, as HSE foram avaliadas por medidas de relato, sendo que $67,5 \%$ deles usaram exclusivamente autorrelato (ou seja, medidas nas quais o participante avaliava seu próprio desempenho), 5,0\% usaram apenas o relato de terceiros e 7,5\% empregaram ambos os tipos de medidas de relato. O relato por terceiros - quer sendo a única forma de avaliação utilizada na pesquisa, quer sendo empregada conjuntamente com instrumentos de autorrelato - foi, em todos os 
casos, realizada por filhos dos cuidadores cujas HSE estavam sendo avaliadas. Tais avaliações foram realizadas com a aplicação do Inventário de Estilos Parentais - IEP (Gomide, 2006) ou Questionário da Qualidade da Interação Familiar na Visão dos Filhos (Cia, D’Affonseca \& Barham, 2004).

Ainda em relação ao relato por terceiros, é interessante notar a ausência de estudos nos quais as HSE de professores fossem avaliadas por seus alunos. No Ensino Superior, por exemplo, estudos têm demonstrado discrepâncias entre a percepção que professores têm do seu desempenho, em relação a diversos aspectos, e a percepção que seus alunos relatam (Carvalho, 1995; Oliveira, Wiles, Fiorin \& Dias, 2014). Instrumentos com evidências de validade que permitam verificar a percepção dos estudantes a respeito do desempenho docente podem ser úteis como feedback da atuação do professor, possibilitando a correção de problemas na relação professor-aluno em si, bem como a correção de problemas de aprendizagem do aluno, decorrentes destas discrepâncias. Isto parece sugerir uma lacuna na área, a qual precisa ser suprida por novos estudos e, provavelmente, pela elaboração de novos instrumentos que permitam a realização de tal tipo de avaliação. Contudo, entende-se que este tipo de avaliação pode apresentar limitações tanto relacionadas a dificuldades do aluno em avaliar o desempenho do professor (por exemplo, relativas à idade do discente, ao tempo de contato deste com o docente, etc.), como à dificuldade prática em obter permissão das escolas para que os alunos avaliem seus professores, o que tem implicações éticas e corporativas.

Cabe destacar que em pelo menos 7,5\% dos estudos as HSE foram avaliadas usando uma combinação de dados obtidos pelo relato de terceiros e por observação. O uso da observação ocorreu de forma diferenciada nos três artigos enquadrados nesta categoria. Rocha, Del Prette e Del Prette (2013), buscando avaliar os efeitos de um programa THS cotidianas e educativas junto a pais de crianças com Transtorno de Déficit de Atenção e Hiperatividade
(TDAH), utilizaram a observação como uma das medidas de avaliação pré e pós-intervenção. A observação foi filmada e a frequência das HSE das mães na interação com a criança, durante atividades livres e orientadas, foi registrada com base no sistema proposto por Z. Del Prette e A. Del Prette (2008). Apesar de não estar descrito na seção de procedimento, no estudo de Coelho e Murta (2007), os autores afirmam que os resultados "foram analisados a partir do relato dos participantes através das entrevistas, da observação clínica e dos escores obtidos pela aplicação do CBCL [Child Behavior Checklist]" (p. 338), porém não apresentam informações adicionais sobre como ocorreu e como foi registrada esta "observação clínica". E no estudo de Garcia-Serpa, Del Prette e Del Prette (2006), cujo objetivo era examinar comportamentos e procedimentos parentais potencialmente favoráveis ao desenvolvimento do repertório pró-social e empático dos filhos, a observação ocorreu em duas situações estruturadas e a videogravação permitiu a análise e categorização de tais comportamentos e procedimentos com base em práticas parentais disponíveis na literatura (suporte, exigência, monitoramento e controle) e em outros tipos de comportamentos identificados a partir da análise da interação pais-filho nas situações estruturadas propostas. Em todos os casos, a observação foi associada a outras medidas de comportamento.

Del Prette e Del Prette (2009) sugerem que os recursos de avaliação das habilidades sociais podem ser divididos em dois grupos: (i) aqueles que avaliam as habilidades sociais de maneira direta, envolvendo tanto a observação por outros em situação natural e estruturada, como a auto-observação e autorregistros fisiológicos; e (ii) aqueles que avaliam as habilidades sociais de forma indireta, os quais incluem a avaliação por relato de outros e por autorrelato. Recursos de avaliação indireta são úteis, dadas à facilidade e rapidez na coleta de dados, quando comparados a recursos de avaliação direta. Todavia, seus resultados devem ser avaliados sem perder de vista as limitações re- 
lativas a esta tipo de medida, tais como o efeito da desejabilidade social (ou seja, responder de acordo com o que é socialmente desejável na situação sobre a qual o desempenho está sendo avaliado e não de acordo com a forma como habitualmente ele ocorre) e problemas relacionados a dificuldades em discriminar situações antecedentes e consequentes, bem como o próprio comportamento. Segundo Del Prette e Del Prette (2009), o ideal, no campo teórico prático das habilidades sociais é, sempre que possível, optar por uma avaliação multimodal, ou seja, que usa mais do que um tipo de medição, pois isso contribui para aumentar a precisão da avaliação realizada.

Outro aspecto que chama atenção quanto à forma como as HSE foram mensuradas é a existência da categoria "Não avaliadas diretamente". Os artigos agrupados nesta categoria referiam-se a estudos que descreviam intervenções realizadas junto à cuidadores primários ou professores cujos efeitos foram avaliados por mudanças que ocorridas no repertório comportamental das crianças (Cia, Barham \& Fontaine, 2010; Lambertucci \& Carvalho, 2008; Pinheiro et al., 2006) ou no repertório de habilidades sociais do professor (Fornazari et al., 2014). Neste último caso, as mudanças comportamentais foram avaliadas por meio da aplicação do Inventário de Habilidades Sociais (IHS-Del-Prette, Del Prette \& Del Prette, 2001), instrumento elaborado para medir as habilidades sociais cotidianas e que, portanto, não focaliza especificamente as HSE. A melhora no repertório de HSE foi inferida a partir da melhora do repertório de habilidades sociais gerais, justificada por Fornazari et al. (2014) como um repertório que poderia favorecer o uso dessas habilidades pelas professoras no contexto de sala de aula nas interações estabelecidas com seus alunos. No entanto, essa é uma suposição que deveria ser objeto de verificação empírica.

Os dados dos estudos empíricos foram coletados principalmente na região sudeste do país, sendo que, das 31 pesquisas realizadas nesta região, 27 ocorreram no estado de São Paulo. Estes dados podem sugerir que o uso da expressão HSE tem se restringido à pesquisas desenvolvidas em determinados centros ou grupos de pesquisa situados prioritariamente nesta região do país. Ao mesmo tempo, aponta para a importância de divulgar o tema e realizar pesquisas que envolvam coletas em outras regiões do país. Não foram localizados estudos sobre HSE fora do Brasil.

Por fim, verificaram-se os instrumentos de autorrelato utilizados, nos estudos empíricos, para mensurar as HSE. Na tabela 2 são apresentados os instrumentos de autorrelato identificados na análise dos 33 artigos que empregaram esta maneira de mensuração (quer como única forma de avaliação, quer acrescida de outras formas de avaliação) e a condição destes instrumentos na literatura.

Quanto à condição dos instrumentos na literatura é possível notar quatro situações distintas: (i) instrumento elaborado para a pesquisa descrita no artigo e que não foi empregado em estudos posteriores, como, por exemplo, o Questionário de avaliação de crenças do Professor - QAC-P utilizado no estudo de Paiva e Del Prette (2009); (ii) instrumentos disponíveis na literatura (porém não normatizados) utilizados por um ou mais estudos, como é o caso do Questionário da qualidade da interação familiar na visão dos pais elaborado por Cia, D'Affonseca e Barham (2004); (iii) instrumentos precursores do RE-HSE-P; e (iv) instrumentos normatizados. Em relação a estas duas últimas condições, é importante lembrar que o processo de elaboração de um instrumento (com vistas à normatização a partir de estudos que garantam suas qualidades psicométricas), tende a ser longo, de tal forma que várias versões podem ser publicadas antes que a versão normatizada esteja finalmente pronta. Este parece ter sido o caso do RE-HSE-P, o qual, até o presente momento, é o único instrumento normatizado para avaliar as HSE-P.

Nos artigos empíricos analisados, foram identificados, no total, 15 instrumentos distintos de autorrelato, dos quais sete foram empregados em um único artigo, quatro relacionavam-se a ins- 
trumentos disponíveis na literatura e utilizados em um ou mais dos artigos analisados, três foram considerados como precursores do RE-HSE-P, e um único instrumento normatizado (RE-HSE-P). Este dado aponta para o fato de que, apesar da diversidade de instrumentos localizados, ainda existe a carência na literatura da área de instrumentos que, além de boas propriedades psicométricas, sejam normatizados. O uso de um instrumento normatizado, além de garantir maior robustez aos dados obtidos em uma pesquisa, também contribui para o constante aprimoramento e levantamento de evidências de confiabilidade e validade do instrumento. Ademais, a elaboração de instrumentos normatizados pode contribuir para alavancar as pesquisas sobre o tema, como pode ser observado no fato de o RE-HSE-P ser o instrumento empregado na maior quantidade de estudos, isto é, em 15 dos 33 artigos que usaram instrumentos de autorrelato para mensurar as HSE (Fantinato \&

Tabela 2

Instrumentos de autorrelato utilizados nos estudos empíricos para mensurar as HSE

\begin{tabular}{|c|c|}
\hline Condição & Instrumento \\
\hline \multirow{7}{*}{$\begin{array}{l}\text { Instrumento elaborado para a pesquisa descrita } \\
\text { no artigo e que não foi empregado em estudos } \\
\text { posteriores }\end{array}$} & $\begin{array}{l}\text { - Avaliação das condições de trabalho e do envolvimento do pai com } \\
\text { seu filho - Versão paterna (Cia \& Barham, 2005) }\end{array}$ \\
\hline & $\begin{array}{l}\text { - Questionário de avaliação de crenças do Professor - QAC-P - (Paiva } \\
\text { \& Del Prette, 2009) }\end{array}$ \\
\hline & - Questionário de HSE (Rocha, Del Prette \& Del Prette, 2013) \\
\hline & $\begin{array}{l}\text { - Questionário sobre HSE maternas, sentimento de insegurança quanto } \\
\text { à educação dos filhos e cuidados fornecidos às crianças (Howat-Rodri- } \\
\text { gues, Tokumaru \& Amorim, 2009) }\end{array}$ \\
\hline & $\begin{array}{l}\text { - Roteiro de entrevista clínica semiestruturada para anamnese de tria- } \\
\text { gem em clínica-escola (Bolsoni-Silva, Paiva \& Barbosa, 2009) }\end{array}$ \\
\hline & $\begin{array}{l}\text { - Roteiro de entrevista estruturada para os pais (Garcia-Serpa, Del Pre- } \\
\text { tte \& Del Prette, 2006) }\end{array}$ \\
\hline & $\begin{array}{l}\text { - Roteiro de entrevista não estruturada para avaliação de pais (Coelho } \\
\text { \& Murta, 2007) }\end{array}$ \\
\hline \multirow{4}{*}{$\begin{array}{l}\text { Instrumentos disponíveis na literatura (porém não } \\
\text { normatizados) utilizados por um ou mais estudos }\end{array}$} & $\begin{array}{l}\text { - Entrevista sobre comportamentos infantis e parentais - E-CIP (Leme, } \\
\text { 2008) }\end{array}$ \\
\hline & $\begin{array}{l}\text { - Questionário da qualidade da interação familiar na visão dos filhos } \\
\text { (Cia, D’Affonseca \& Barham, 2004) }\end{array}$ \\
\hline & $\begin{array}{l}\text { - Questionário da qualidade da interação familiar na visão dos pais } \\
\text { (Cia, D’Affonseca \& Barham, 2004) }\end{array}$ \\
\hline & $\begin{array}{l}\text { - Questionário de habilidades sociais educativas para professores (Al- } \\
\text { meida-Verdu, 2008) }\end{array}$ \\
\hline \multirow{3}{*}{ Instrumentos precursores do RE-HSE-P } & $\begin{array}{l}\text { - Questionário de habilidades sociais educativas parentais (QHSE-P, } \\
\text { adaptação de Bolsoni-Silva, 2003) }\end{array}$ \\
\hline & - Questionário elaborado a partir dos dados obtidos por Silva (1997) \\
\hline & $\begin{array}{l}\text { - Roteiro de entrevista elaborado para a pesquisa (Bolsoni-Silva \& Del } \\
\text { Prette, 2002) }\end{array}$ \\
\hline Instrumentos normatizados & $\begin{array}{l}\text { Roteiro de Entrevista de Habilidades Sociais Educativas Parentais - } \\
\text { RE-HSE-P (Bolsoni-Silva, Loureiro \& Marturano, 2011) }\end{array}$ \\
\hline
\end{tabular}


Cia, 2015; Sabbag \& Bolsoni-Silva, 2015; Bolsoni-Silva \& Mariano, 2014; Fantinato \& Cia, 2014a; Bolsoni-Silva, Mariano, Loureiro \& Bonaccorsi, 2013; Bolsoni-Silva \& Borelli, 2012; Bolsoni-Silva $\&$ Loureiro, 2011; Sabbag \& Bolsoni-Silva, 2011; Boas \& Bolsoni-Silva, 2010; Bolsoni-Silva \& Loureiro, 2010; Bolsoni-Silva, Marturano, et al., 2010; Bolsoni-Silva, Rodrigues, et al., 2010; Bolsoni-Silva \& Marturano, 2008; Bolsoni-Silva, Silveira \& Ribeiro, 2008; Bolsoni-Silva, Silveira \& Marturano, 2008).

Por fim, em concordância com os outros achados descritos acima, a análise dos títulos dos instrumentos permite observar que, em sua maioria, os instrumentos estão voltados para avaliar HSE na interação pais/cuidadores-filhos/crianças. Apenas dois deles estão voltados especificamente para a relação professor-aluno, a saber: o Questionário de avaliação de crenças do Professor (QAC-P), elaborado por Paiva e Del Prette (2009), com base na literatura da área, e o Questionário de habilidades sociais educativas para professores, elaborado por Almeida-Verdu (2008), a partir do RE-HSE-P proposto por Bolsoni-Silva (2009). Portanto, parece haver uma lacuna que precisa ser suprida, dado o papel relevante que professores assumem no processo de desenvolvimento do repertório de habilidades sociais de seus alunos.

\section{Considerações Finais}

O presente trabalho teve como objetivos mapear a produção brasileira que tem enfocado as HSE, identificar os tipos de estudos que têm sido desenvolvidos sobre o tema e características desses estudos. A análise do corpus permite destacar alguns pontos dessa produção acadêmica.

$\mathrm{O}$ primeiro refere-se ao sentido atribuído à expressão HSE. Esta expressão surge no bojo do campo teórico prático das habilidades sociais e, a partir dos artigos analisados, é possível observar três situações distintas quanto ao uso de sua definição: adoção da definição mais ampla, tal como proposta por Del Prette e Del Prette (2010/2001); adoção do termo HSE-P, tal como aplicado à população específica de pais, conforme Silva (2000); uso da noção de HSE, mas sem uma definição explícita. A maioria dos estudos estava voltada para a relação educativa pais-filhos, o que contribuiu para a grande quantidade de artigos que utilizaram a expressão HSE-P. Cabe ressaltar, conforme sugerem Garcia, Del Prette e Del Prette (2006), que estudos que abordam comportamentos dos pais em relação aos filhos utilizam diversas expressões para se referirem a esses comportamentos, tais como práticas educativas, estilos parentais, envolvimento, etc., e "poucos deles fazem referência às unidades comportamentais menores associadas ou características de cada uma dessas classes" (p. 78), que levariam às HSE. Esta situação pode contribuir para o uso indiscriminado da expressão HSE, sem uma definição precisa e adequada.

Em segundo lugar, publicações acerca deste tema parecem girar em torno de determinados centros ou grupos de pesquisa, o que pode ser observado tanto por meio dos autores que se destacam em quantidade de artigos publicados sobre o tema, como pela região do país na qual a maioria das coletas de dados foi realizada. Também foi possível verificar uma quantidade bem maior de estudos empíricos em comparação com teóricos. Enquanto estes buscavam elucidar, principalmente, questões relativas às HSE e a inclusão de portadores de necessidades educacionais especiais, aqueles, em sua maioria, tinham como objetivo validar instrumentos, avaliar as HSE e/ou compará-las a outras variáveis.

Outro ponto que se destaca é que, na maioria dos estudos empíricos, os participantes foram cuidadores primários. Pouquíssimos foram os estudos nos quais outros tipos de agentes educativos foram envolvidos. Ainda chama a atenção o fato de que as HSE foram mensuradas, prioritariamente, através de recursos indiretos de avaliação, tais como instrumentos de autorrelato ou de relato por terceiros. Os instrumentos de autorrelato foram os mais 
utilizados, quer como único recurso de avaliação das HSE, quer acompanhados por outras medidas (avaliação por terceiros ou observação). Nessa direção, o uso de recursos diretos de avaliação das habilidades sociais - por exemplo, observação - foi empregado em um número reduzido de estudos, de forma distinta em cada estudo e como medida complementar ao uso de recursos indiretos de avaliação. Apesar do número expressivo de instrumentos de autorrelato identificados entre os artigos analisados, foi possível observar que o instrumento mais empregado foi o RE-HSE-P, em associação com o processo de validação desse instrumento (Bolsoni-Silva, 2008; Bolsoni-Silva, 2009; Bolsoni-Silva \& Loureiro, 2010) e, possivelmente por isso, utilizado, até o momento, basicamente nos estudos conduzidos pelos seus autores. Porém também se observou estudos sobre relação pais-filhos que usaram outros instrumentos e procedimentos.

Ainda em relação à mensuração das HSE, apesar de não aparecer neste corpus, existem instrumentos em processo de elaboração que buscam avaliar as HSE tomando como base a definição mais ampla proposta por Z. Del Prette e A. Del Prette (2008). São eles: Inventário de Habilidades Sociais Educativas versão para pais - IHSE-Pais (Del Prette \& Del Prette, 2013a), Inventário de Habilidades Sociais Educativas para professores do ensino fundamental - IHSE-Prof (Del Prette \& Del Prette, 2013b) e o Inventário de Habilidades Sociais Educativas para Professores Universitários - IHSE-PU (Del Prette \& Del Prette, 2013c). Os dois primeiros, já dispõem de qualidades psicométricas iniciais e estão sendo empregados por pesquisadores de diversas partes do país, aparecendo inicialmente em resumos de trabalhos publicados em anais de eventos (por exemplo, Brasil \& Cia, 2013; Fantinato \& Cia, 2014b; Leme \& Fernandes, 2015; Moreira, Del Prette \& Dias, 2013, Rosin-Pinola, 2015). O IHSE-PU ainda encontra-se em fase de coleta e análise de dados visando aferir suas qualidades psicométricas.
Por fim, cabe indicar que o sistema de HSE-P, proposto por Bolsoni-Silva (2008) para a relação parental tem sido utilizado pela autora também para avaliar as práticas educativas de professores na interação com seus alunos (Silva, Bolsoni-Silva, Rodrigues \& Capellini, 2015; Bolsoni-Silva \& Mariano, 2014). Certamente, mais pesquisas serão necessárias para avaliar em que medida as categorias propostas para pais são suficientes para contemplar as diversas demandas existentes na relação professor-aluno.

A análise do corpus deste estudo permitiu identificar diversas lacunas sobre o tema que apontam para novas pesquisas sobre as HSE. Algumas delas foram: possibilidade de ampliar o uso da expressão HSE, incentivando pesquisas sobre o tema em outros ambientes educativos, além daqueles que os atuais estudos têm focalizado; estudos teóricos sobre as HSE relacionados principalmente à área de Educação Especial na direção do investimento feito por A. Del Prette e Z. Del Prette (2008) e Rosin-Pinola e Del Prette (2014); poucos instrumentos já normatizados na área das HSE, apesar das iniciativas nesse sentido; estudos sobre as HSE junto a outros educadores (que não os cuidadores primários ou professores da educação básica).

As conclusões finais do presente estudo se limitam ao corpus analisado, o qual foi composto apenas por artigos publicados em periódicos científicos. Novas pesquisas que incluam outros tipos de publicações - tais como dissertações, teses, capítulos de livros e trabalhos publicados em anais de eventos - podem trazer mais informações a respeito de como as HSE tem sido tratadas na literatura, além de contribuir para o incentivo de novas pesquisas a respeito do tema.

\section{Referências}

Almeida-Verdu, A. C. (2008). Repensando a avaliação. Em V. L. M. F. Capellini (Org.). Práticas em educação especial e inclusiva na área 
da deficiência mental - Volume 12. Bauru, SP: $\mathrm{MEC} / \mathrm{FC} / \mathrm{SEE}$.

Boas, A. C. V. B. V., \& Bolsoni-Silva, A. T. (2010). Habilidades sociais educativas de mães separadas e sua relação com o comportamento de pré-escolares. Psico-USF, 15(3), 301-310. doi: http://dx. doi.org/10.1590/S1413-82712010000300004

Bolsoni-Silva, A. T. (2009). Estudos de confiabilidade e de validade do roteiro de entrevista de habilidades sociais educativas parentais (REHSE-P). (Tese de Pós-Doutorado, Universidade de São Paulo, Brasil, Faculdade de Medicina, Universidade de São Paulo, Ribeirão Preto).

Bolsoni-Silva, A. T. (2003). Habilidades Sociais Educativas, variáveis contextuais e problemas de comportamento: comparando pais e mães de pré-escolares (Tese de Doutorado, Universidade de São Paulo, Ribeirão Preto).

Bolsoni-Silva, A. T. (2008). Roteiro de Entrevista de Habilidades Sociais Educativas Parentais (REHSE-P): Categorias e testagem preliminares. Em L. D. Weber (Org.), Família e Desenvolvimento - Visões Interdisciplinares (pp. 145-158). Curitiba: Juruá.

Bolsoni-Silva, A. T., \& Borelli, L. M. (2012). Treinamento de Habilidades Sociais Educativas Parentais: Comparação de Procedimentos a partir do Tempo de Intervenção. Estudos e Pesquisas em Psicologia, 12(1), 36-58.

Bolsoni-Silva, A. T., \& Del Prette, A. (2002). O que os pais falam sobre suas habilidades sociais e de seus filhos? Argumento, 3(7), 71-86.

Bolsoni-Silva, A. T., \& Loureiro, S. R. (2011). Práticas educativas parentais e repertório comportamental infantil: comparando crianças diferenciadas pelo comportamento. Paidéia (Ribeirão Preto), 21(48), 61-71. doi: http://dx. doi.org/10.1590/S0103-863X2011000100008

Bolsoni-Silva, A. T., \& Loureiro, S. R. (2010). Validação do Roteiro de Entrevista de Habilidades Sociais Educativas Parentais (RE-HSE-P). Avaliação Psicológica, 9(1), 63-75.
Bolsoni-Silva, A. T., Loureiro, S. R., \& Marturano, E. M. (2011). Roteiro de Entrevista de Habilidades Sociais Educativas Parentais (RE-HSE-P). Manual Técnico. São Paulo: Editora Vetor.

Bolsoni-Silva, A. T., \& Mariano, M. L. (2014). Práticas educativas de professores e comportamentos infantis, na transição ao primeiro ano do Ensino Fundamental. Estudos e Pesquisas em Psicologia, 14(3), 814-833.

Bolsoni-Silva, A. T., Mariano, M. L., Loureiro, S. R., \& Bonaccorsi, C. (2013). Contexto escolar: práticas educativas do professor, comportamento e habilidades sociais infantis. Psicologia Escolar e Educacional, 17(2), 259-269. doi: http://dx. doi.org/10.1590/S1413-85572013000200008

Bolsoni-Silva, A. T., \& Marturano, E. M. (2008). Habilidades sociais educativas parentais e problemas de comportamento: comparando pais e mães de pré-escolares. Aletheia, 27, 126-138.

Bolsoni-Silva, A. T., Marturano, E. M., Barbosa, C. G., Paiva, M. M., Costa, N. L., \& Santos, L. C. (2010). Práticas parentais e repertório infantil: caracterização da demanda por atendimento e predição de abandono. Aletheia, 32, 121-133.

Bolsoni-Silva, A. T., Paiva, M. M., \& Barbosa, C. G. (2009). Problemas de comportamento de crianças/adolescentes e dificuldades de pais/ cuidadores: um estudo de caracterização. Psicologia Clínica, 21(1), 169-184. doi: http://dx. doi.org/10.1590/S0103-56652009000100012

Bolsoni-Silva, A. T., Rodrigues, O. M. P. R., Abramides, D. V. M., Souza, L. S., \& Loureiro, S. R. (2010). Práticas educativas parentais de crianças com deficiência auditiva e de linguagem. Revista Brasileira de Educação Especial, 16(2), 265-282. doi: http://dx.doi.org/10.1590/ S1413-65382010000200008

Bolsoni-Silva, A. T., Silveira, F. F., \& Marturano, E. M. (2008). Promovendo habilidades sociais educativas parentais na prevenção de problemas de comportamento. Revista Brasileira de Terapia Comportamental e Cognitiva, 10(2), 125-142. 
Bolsoni-Silva, A. T., Silveira, F. F., \& Ribeiro, D. C. (2008). Avaliação dos efeitos de uma intervenção com mães/cuidadoras: contribuições do treinamento em habilidades sociais. Contextos Clínicos, 1(1), 19-27.

Brasil, S. E. R., \& Cia, F. (2013). Pais e filhos: habilidades sociais educativas parentais e habilidades sociais infantis. Anais do VIII Encontro da Associação Brasileira de Pesquisadores em Educação Especial, Londrina, Paraná.

Carvalho, M. A. V. (1995). Relação professor-aluno: fatores intervenientes tendo em vista a aprendizagem. Semina: Ciências Sociais e Humanas, 16(2), 57-65. doi: http://dx.doi.org/10.5433/1 679-0383.1995v16n3p57

Cia, F., \& Barham, E. J. (2005). A relação entre o turno de trabalho do pai e o autoconceito do filho. Psico, 36(1), 29-35.

Cia, F., Barham, E. J., \& Fontaine, A. M. G. V. (2010). Impactos de uma intervenção com pais: o desempenho acadêmico e comportamento das crianças na escola. Psicologia: Reflexão e Crítica, 23(3), 533-543. doi: http://dx.doi. org/10.1590/S0102-79722010000300014

Cia, F., D'Affonseca, S. M., \& Barham, E. J. (2004). A relação entre o envolvimento paterno e o desempenho acadêmico dos filhos. Paidéia (Ribeirão Preto), 14(29), 277-286. doi: http://dx. doi.org/10.1590/S0103-863X2004000300004

Cia, F., Pereira, C. S., Del Prette, Z. A. P., \& Del Prette, A. (2007). Habilidades sociais das mães e envolvimento com os filhos: um estudo correlacional. Estudos de Psicologia (Campinas), 24(1), 3-11. doi: http://dx.doi.org/10.1590/ S0103-166X2007000100001

Coelho, M. V., \& Murta, S. G. (2007). Treinamento de pais em grupo: um relato de experiência. Estudos de Psicologia (Campinas), 24(3), 333-341.

Del Prette, A., \& Del Prette, Z. A. P. (2008). Pais e professores contribuindo para o processo de inclusão: Que habilidades sociais educativas devem apresentar? Em E. G. Mendes, M. A. Almeida \& M. C. P. I. Hayashi (Orgs.), Temas em Educação Especial: Conhecimentos para fundamentar a prática (pp.239-256). Araraquara: Junqueira e Marin.

Del Prette, A., Del Prette, Z. A. P., Pontes, A. C., \& Torres, A. C. (a). Efeitos de um programa de intervenção sobre aspectos topográficos das habilidades sociais de professores. Psicologia Escolar e Educacional, 2(1), 11-22. doi: http://dx. doi.org/10.1590/S1413-85571998000100002

Del Prette, Z. A. P., \& Del Prette, A. (2013a). Inventário de habilidades sociais educativas - versão Pais (IHSE-Pais): Dados psicométricos preliminares. Relatório não publicado disponível com os autores

Del Prette, Z. A. P., \& Del Prette, A. (2013b). Inventário de habilidades sociais educativas - versão professor do ensino fundamental (IHSE-Prof): Dados psicométricos preliminares. Relatório não publicado disponível com os autores.

Del Prette, Z. A. P., \& Del Prette, A. (2013c). Inventário de habilidades sociais educativas - versão professor universitário (IHSE-PU): Estudos psicométricos em andamento. Texto disponível com os autores.

Del Prette, Z. A. P. \& Del Prette, A. (2010). Psicologia das relações interpessoais: vivencias para o trabalho em grupo - 8. ${ }^{\text {a }}$ ed. - . Petrópolis, RJ: Vozes. Primeira edição publicada em 2001.

Del Prette, Z. A. P., \& Del Prette, A. (2009). Avaliação de habilidades sociais: bases conceituais, instrumentos e procedimentos. Em Z.A. P Del Prette e A. Del Prette (Orgs.), Psicologia das habilidades sociais: diversidade teórica e suas implicações (pp. 189-231). Petrópolis, RJ: Vozes.

Del Prette, Z. A. P., \& Del Prette, A. (2008). Um sistema de categorias de habilidades sociais educativas. Paidéia (Ribeirão Preto), 18(41), 517-530. doi: http://dx.doi.org/10.1590/S0103863X2008000300008

Del Prette, Z. A. P., \& Del Prette, A. (2001). Inventário de Habilidades Sociais (IHS-Del Prette): 
Manual de aplicação e interpretação. São Paulo: Casa do Psicólogo.

Del Prette, Z. A. P., \& Del Prette, A. (1997). Um programa de desenvolvimento de habilidades sociais na formação continuada de professores. Em Associação Nacional de Pesquisa Em Educação (Org.), CD-Rom dos trabalhos selecionados para apresentação (29 p.): 20a. Caxambu MG: Reunião Anual da ANPED.

Del Prette, Z. A. P., Del Prette, A., Garcia, F. A Silva, A. B. T., \& Puntel, L. (1998). Habilidades sociais do professor: Um estudo de caso. Psicologia: Reflexão e Crítica, 11(3), 611623. doi: http://dx.doi.org/10.1590/S010279721998000300016

Fantinato, A. C., \& Cia, F. (2015). Habilidades sociais educativas, relacionamento conjugal e comportamento infantil na visão paterna: um estudo correlacional. Psico (Porto Alegre), 46(1), 120-128. doi: http://dx.doi.org/10.15448/19808623.2015.1.17330

Fantinato, A. C., \& Cia, F. (2014a). Habilidades sociais educativas paternas e comportamento infantil. Psicologia Argumento, 32(supl.1), 177186. doi: 10.7213/psicol.argum.32.S01.AO16

Fantinato, A. C., \& Cia, F. (2014b). Problemas de comportamento de pré-escolares e habilidades sociais educativas de seus pais. Anais do $11^{\circ}$ Encontro de Pesquisa em Educação da Região Sudeste: culturas, políticas e práticas pedagógicas e suas relações com a pesquisa - Volume I (pp. 2404-2413). São João del-Rei: Programa de Pós-Graduação em Processos Socioeducativos e Práticas Escolares, Universidade Federal de São João del-Rei. Recuperado de http://www. anpedsudeste2014.com.br/

Fornazari, S. A., Kienen, N., Vila, E. M., Nantes, F. O., \& Proença, M. R. (2014). Programa informatizado para capacitar professores em habilidades sociais: contribuições para a inclusão. Psicologia da Educação, (38), 17-34.

Galvão, T. F., Pansani, T. S. A., \& Harrad, D. (2015). Principais itens para relatar Revisões sistemáticas e Meta-análises: A recomendação PRISMA. Epidemiologia e Serviços de Saúde, 24(2), 335-342. doi: https://dx.doi.org/10.5123/ S1679-49742015000200017

Garcia-Serpa, F. A., Del Prette, Z. A. P., \& Del Prette, A. (2006). Meninos pré-escolares empáticos e não-empáticos: empatia e procedimentos educativos dos pais. Interamerican Journal of Psychology, 40(1), 73-84.

Gomide, P. I. C. (2006). Manual do inventário de estilos parentais: modelo teórico, manual de aplicação, apuração e interpretação. Petrópolis, RJ: Vozes.

Gomide, P. I. C. (2003). Estilos parentais e comportamento anti-social. Em A. Del Prette \& Z. A. P. Del Prette (Orgs), Habilidades sociais, desenvolvimento e aprendizagem (pp. 21-60). Campinas, SP: Alínea.

Howat-Rodrigues, A. B. C., Tokumaru, R. S., \& Amorim, T. N. (2009). Mães adotivas e genéticas: habilidades, insegurança e apoio percebido. Psico (Porto Alegre), 40(2), 202-209.

Lambertucci, M. R., \& Carvalho, H. W. (2008). Avaliação da efetividade terapêutica de um programa de treinamento de pais em uma comunidade carente de Belo Horizonte. Contextos Clínicos, 1(2), 106-112.

Leme, V. B. R., \& Fernandes, L. M. (2015). O impacto de recursos pessoais e contextuais no desempenho escolar de alunos na transição para o ensino médio. Anais do VSeminário Internacional de Habilidades Sociais - Habilidades sociais e relações interpessoais: interfaces conceituais, metodológicas e aplicadas (pp. 54-55). Recuperado de http://www.sihs.ufscar.br/.

Leme, V. R. B. (2008). Práticas educativas maternas e a sua relação com comportamentos habilidosos e problemas de comportamento de pré-escolares. (Dissertação de Mestrado, Universidade Estadual Paulista, Bauru).

Manolio, C. L. (2009). Análise das habilidades sociais educativas na interação professor-aluno 
(Dissertação de Mestrado, Universidade Federal de São Carlos, São Carlos).

Moreira, L. B.; Del Prette, Z. A. P., \& Dias, T. P. (2013). Habilidades sociais do professor e comportamento social do aluno. Anais do IV Seminário Internacional de Habilidades Sociais: diálogos e intercâmbios sobre pesquisa e prática (pp. 49-50). Niterói, RJ. Recuperado de http://www.rihs.ufscar.br/iv-sihs/.

Oliveira, C. T., Wiles, J. M., Fiorin, P. C., \& Dias, A. C. G. (2014). Percepções de estudantes universitários sobre a relação professor-aluno. Psicologia Escolar e Educacional, 18(2), 239-246. doi: http://dx.doi.org/10.1590/21753539/2014/0182739

Paiva, M. L. M. F., \& Del Prette, Z. A. P. (2009). Crenças docentes e implicações para o processo de ensino-aprendizagem. Psicologia Escolar e Educacional, 13(1), 75-85.

Rocha, M. M., \& Del Prette, Z. A. P. (2010). Habilidades sociais educativas para mães de crianças com TDAH e a inclusão escolar. Psicologia Argumento, 28(60), 31-41.

Rocha, M. M., Andrade, P., \& Dourado, T. L. S. (2011). Comportamentos-alvos em programas de habilidades sociais educativas para pais. Revista Educação Especial, 24(41), 453-465. doi: http://dx.doi.org/10.5902/1984686X3579

Rocha, M. M., Del Prette, Z. A. P., \& Del Prette, A. (2013). Avaliação de um Programa de Habilidades Sociais Educativas para mães de crianças com TDAH. Acta Comportamentalia, 21(3), 273-283.

Rosin-Pinola, A. R. (2015). Habilidades sociais educativas junto a professores de escolas públicas. Anais do V Seminário Internacional de
Habilidades Sociais - Habilidades sociais e relações interpessoais: interfaces conceituais, metodológicas e aplicadas (pp. 57). Recuperado de http://www.sihs.ufscar.br/.

Rosin-Pinola, A. R., \& Del Prette, Z. A. P. (2014). Inclusão escolar, formação de professores e a assessoria baseada em habilidades sociais educativas. Revista Brasileira de Educação Especial, 20(3), 341-356. doi: http://dx.doi. org/10.1590/S1413-65382014000300003

Sabbag, G. M., \& Bolsoni-Silva, A. T. (2015). Interações entre mães e adolescentes e os problemas de comportamento. Arquivos Brasileiros de Psicologia, 67(1), 68-83.

Sabbag, G. M., \& Bolsoni-Silva, A. T. (2011). A relação das Habilidades Sociais educativas e das práticas educativas maternas com os problemas de comportamento em adolescentes. Estudos e Pesquisas em Psicologia, 11(2), 423-441.

Silva, A. T. B. (2000). Problemas de comportamento e comportamentos socialmente adequados: sua relação com as habilidades sociais educativas de pais (Dissertação de Mestrado, Universidade Federal de São Carlos, São Carlos).

Silva, N. R., Bolsoni-Silva, A. T., Rodrigues, O. M. P. R., \& Capellini, V. L. M. F. (2015). O trabalho do professor, indicadores de Burnout, práticas educativas e comportamento dos alunos: correlação e predição. Revista Brasileira de Educação Especial, 21(3), 363-376. doi: http://dx. doi.org/10.1590/S1413-65382115000300004

Von Hoendorff, J. (2014). Como escrever um artigo de revisão de literatura. Em S. H. Koller, M. C. P. P. Couto e J. Von Hoendorff(Orgs.), Manual de produção científica. Porto Alegre: Penso.

\section{Recebido: Agosto 10, 2016 Aprovado: Dezembro 27, 2016}




\section{Apêndice - Referências dos artigos que compuseram o corpus desta pesquisa}

Boas, A. C. V. B. V., \& Bolsoni-Silva, A. T. (2010). Habilidades sociais educativas de mães separadas e sua relação com o comportamento de pré-escolares. Psico-USF, 15(3), 301-310. doi: http://dx.doi. org/10.1590/S1413-82712010000300004

Bolsoni-Silva, A. T., \& Borelli, L. M. (2012). Treinamento de Habilidades Sociais Educativas Parentais: Comparação de Procedimentos a partir do Tempo de Intervenção. Estudos e Pesquisas em Psicologia, 12(1), 36-58.

Bolsoni-Silva, A. T., \& Del Prette, A. (2002). O que os pais falam sobre suas habilidades sociais e de seus filhos? Argumento, 3(7), 71-86.

Bolsoni-Silva, A. T., \& Loureiro, S. R. (2010). Validação do Roteiro de Entrevista de Habilidades Sociais Educativas Parentais (RE-HSE-P). Avaliação Psicológica, 9(1), 63-75.

Bolsoni-Silva, A. T., \& Loureiro, S. R. (2011). Práticas educativas parentais e repertório comportamental infantil: comparando crianças diferenciadas pelo comportamento. Paidéia (Ribeirão Preto), 21(48), 61-71. doi: http://dx.doi.org/10.1590/S0103-863X2011000100008

Bolsoni-Silva, A. T., \& Mariano, M. L. (2014). Práticas educativas de professores e comportamentos infantis, na transição ao primeiro ano do Ensino Fundamental. Estudos e Pesquisas em Psicologia, 14(3), 814-833.

Bolsoni-Silva, A. T., \& Marturano, E. M. (2002). Práticas educativas e problemas de comportamento: uma análise à luz das habilidades sociais. Estudos de Psicologia (Natal), 7(2), 227-235. doi: http:// dx.doi.org/10.1590/S1413-294X2002000200004

Bolsoni-Silva, A. T., \& Marturano, E. M. (2007). A qualidade da interação positiva e da consistência parental na sua relação com problemas de comportamentos de pré-escolares. Interamerican Journal of Psychology, 41(3), 349-358.

Bolsoni-Silva, A. T., \& Marturano, E. M. (2008). Habilidades sociais educativas parentais e problemas de comportamento: comparando pais e mães de pré-escolares. Aletheia, 27, 126-138.

Bolsoni-Silva, A. T., Del Prette, A., \& Oishi, J. (2003). Habilidades sociais de pais e problemas de comportamento de filhos. Argumento, 9, 11-29.

Bolsoni-Silva, A. T. Mariano, M. L., Loureiro, S. R., \& Bonaccorsi, C. (2013). Contexto escolar: práticas educativas do professor, comportamento e habilidades sociais infantis. Psicologia Escolar e Educacional, 17(2), 259-269. doi: http://dx.doi.org/10.1590/S1413-85572013000200008

Bolsoni-Silva, A. T., Mariano, M. L., Loureiro, S. R., \& Bonaccorsi, C. (2013). Contexto escolar: práticas educativas do professor, comportamento e habilidades sociais infantis. Psicologia Escolar e Educacional, 17(2), 259-269. doi: http://dx.doi.org/10.1590/S1413-85572013000200008

Bolsoni-Silva, A. T., Marturano, E. M., Barbosa, C. G., Paiva, M. M., Costa, N. L., \& Santos, L. C. (2010). Práticas parentais e repertório infantil: caracterização da demanda por atendimento e predição de abandono. Aletheia, 32, 121-133.

Bolsoni-Silva, A. T., Paiva, M. M., \& Barbosa, C. G. (2009). Problemas de comportamento de crianças/ adolescentes e dificuldades de pais/cuidadores: um estudo de caracterização. Psicologia Clínica, 21(1), 169-184. doi: http://dx.doi.org/10.1590/S0103-56652009000100012

Bolsoni-Silva, A. T., Rodrigues, O. M. P. R., Abramides, D. V. M., Souza, L. S., \& Loureiro, S. R. (2010). Práticas educativas parentais de crianças com deficiência auditiva e de linguagem. Revista Brasileira de Educação Especial, 16(2), 265-282. doi: http://dx.doi.org/10.1590/S1413-65382010000200008 
Bolsoni-Silva, A. T., Salina-Brandão, A., Versuti-Stoque, F. M., \& Rosin-Pinola, A. R. (2008). Avaliação de um programa de intervenção de habilidades sociais educativas parentais: um estudo-piloto. Psicologia: Ciência e Profissão, 28(1), 18-33. doi: http://dx.doi.org/10.1590/S1414-98932008000100003

Bolsoni-Silva, A. T., Silveira, F. F., \& Marturano, E. M. (2008). Promovendo habilidades sociais educativas parentais na prevenção de problemas de comportamento. Revista Brasileira de Terapia Comportamental e Cognitiva, 10(2), 125-142.

Bolsoni-Silva, A. T., Silveira, F. F., \& Ribeiro, D. C. (2008). Avaliação dos efeitos de uma intervenção com mães/cuidadoras: contribuições do treinamento em habilidades sociais. Contextos Clínicos, 1(1), 19-27.

Cardozo, A., \& Soares, A. B. (2010). A influência das habilidades sociais no envolvimento de mães e pais com filhos com retardo mental. Aletheia, (31), 39-53.

Cia, F., \& Barham, E. J. (2005). A relação entre o turno de trabalho do pai e o autoconceito do filho. Psico, 36(1), 29-35.

Cia, F., Barham, E. J., \& Fontaine, A. M. G. V. (2010). Impactos de uma intervenção com pais: o desempenho acadêmico e comportamento das crianças na escola. Psicologia: Reflexão e Crítica, 23(3), 533-543. doi: http://dx.doi.org/10.1590/S0102-79722010000300014

Cia, F., D’Affonseca, S. M., \& Barham, E. J. (2004). A relação entre o envolvimento paterno e o desempenho acadêmico dos filhos. Paidéia (Ribeirão Preto), 14(29), 277-286. doi: http://dx.doi. org/10.1590/S0103-863X2004000300004

Cia, F., Pamplin, R. C. O., \& Del Prette, Z. A. P. (2006). Comunicação e participação pais-filhos: correlação com habilidades sociais e problemas de comportamento dos filhos. Paidéia (Ribeirão Preto), 16(35), 395-406. doi: http://dx.doi.org/10.1590/S0103-863X2006000300010

Cia, F., Pamplin, R. C. O., \& Williams, L. C. A. (2008). O impacto do envolvimento parental no desempenho acadêmico de crianças escolares. Psicologia em Estudo, 13(2), 351-360. doi: http://dx.doi. org/10.1590/S1413-73722008000200018

Cia, F., Pereira, C. S., Del Prette, Z. A. P., \& Del Prette, A. (2007). Habilidades sociais das mães e envolvimento com os filhos: um estudo correlacional. Estudos de Psicologia (Campinas), 24(1), 3-11. doi: http://dx.doi.org/10.1590/S0103-166X2007000100001

Cia, F., Pereira, C. S., Del Prette, Z. A. P., \& Del Prette, A. (2006). Habilidades sociais parentais e o relacionamento entre pais e filhos. Psicologia em Estudo, 11(1), 73-81.

Coelho, M. V., \& Murta, S. G. (2007). Treinamento de pais em grupo: um relato de experiência. Estudos de Psicologia (Campinas), 24(3), 333-341.

Del Prette, Z. A. P., \& Del Prette, A. (2008). Um sistema de categorias de habilidades sociais educativas. Paidéia (Ribeirão Preto), 18(41), 517-530. doi: http://dx.doi.org/10.1590/S0103-863X2008000300008

Fantinato, A. C., \& Cia, F. (2014). Habilidades sociais educativas paternas e comportamento infantil. Psicologia Argumento, 32(supl.1), 177-186. doi: 10.7213/psicol.argum.32.S01.AO16

Fantinato, A. C., \& Cia, F. (2015). Habilidades sociais educativas, relacionamento conjugal e comportamento infantil na visão paterna: um estudo correlacional. Psico (Porto Alegre), 46(1), 120-128. doi: http://dx.doi.org/10.15448/1980-8623.2015.1.17330

Fornazari, S. A., Kienen, N., Vila, E. M., Nantes, F. O., \& Proença, M. R. (2014). Programa informatizado para capacitar professores em habilidades sociais: contribuições para a inclusão. Psicologia da Educação, (38), 17-34. 
Freitas, M. G., Del Prette, Z. A. P., \& Del Prette, A. (2007). Melhorando habilidades sociais de crianças com deficiência visual: Um programa de intervenção para mães. Revista Benjamin Constant, 13(37), 17-27.

Freitas, M. G., Del Prette, Z. A. P., \& Del Prette, A. (2007). Melhorando habilidades sociais de crianças com deficiência visual: Um programa de intervenção para mães. Revista Benjamin Constant, 13(37), 17-27.

Garcia-Serpa, F. A., Del Prette, Z. A. P., \& Del Prette, A. (2006). Meninos pré-escolares empáticos e não-empáticos: empatia e procedimentos educativos dos pais. Interamerican Journal of Psychology, 40(1), 73-84.

Howat-Rodrigues, A. B. C., Tokumaru, R. S., \& Amorim, T. N. (2009). Mães adotivas e genéticas: habilidades, insegurança e apoio percebido. Psico (Porto Alegre), 40(2), 202-209.

Lambertucci, M. R., \& Carvalho, H. W. (2008). Avaliação da efetividade terapêutica de um programa de treinamento de pais em uma comunidade carente de Belo Horizonte. Contextos Clínicos, 1(2), 106-112.

Leme, V. B. R., \& Bolsoni-Silva, A. T. (2010a). Habilidades Sociais Educativas Parentais e comportamentos de pré-escolares. Estudos de Psicologia (Natal), 15(2), 161-173. doi: http://dx.doi.org/10.1590/ S1413-294X2010000200005

Leme, V. B. R., \& Bolsoni-Silva, A. T. (2010b). Habilidades sociais e problemas de comportamento: um estudo exploratório baseado no modelo construcional. Aletheia, (31), 149-167.

Paiva, M. L. M. F., \& Del Prette, Z. A. P. (2009). Crenças docentes e implicações para o processo de ensino-aprendizagem. Psicologia Escolar e Educacional, 13(1), 75-85.

Pinheiro, M. I. S., Haase, V. G., Del Prette, A., Amarante, C. L. D., \& Del Prette, Z. A. P. (2006). Treinamento de habilidades sociais educativas para pais de crianças com problemas de comportamento. Psicologia: Reflexão e Crítica, 19(3), 407-414.

Rocha, M. M., \& Del Prette, Z. A. P. (2010). Habilidades sociais educativas para mães de crianças com TDAH e a inclusão escolar. Psicologia Argumento, 28(60), 31-41.

Rocha, M. M., Andrade, P., \& Dourado, T. L. S. (2011). Comportamentos-alvos em programas de habilidades sociais educativas para pais. Revista Educação Especial, 24(41), 453-465. doi: http://dx.doi. org/10.5902/1984686X3579

Rocha, M. M., Del Prette, Z. A. P., \& Del Prette, A. (2013). Avaliação de um Programa de Habilidades Sociais Educativas para mães de crianças com tDAH. Acta Comportamentalia, 21(3), 273-283.

Rosin-Pinola, A. R., \& Del Prette, Z. A. P. (2014). Inclusão escolar, formação de professores e a assessoria baseada em habilidades sociais educativas. Revista Brasileira de Educação Especial, 20(3), 341-356. doi: http://dx.doi.org/10.1590/S1413-65382014000300003

Sabbag, G. M., \& Bolsoni-Silva, A. T. (2011). A relação das Habilidades Sociais educativas e das práticas educativas maternas com os problemas de comportamento em adolescentes. Estudos e Pesquisas em Psicologia, 11(2), 423-441.

Sabbag, G. M., \& Bolsoni-Silva, A. T. (2015). Interações entre mães e adolescentes e os problemas de comportamento. Arquivos Brasileiros de Psicologia, 67(1), 68-83.

Silva, N. R., Bolsoni-Silva, A. T., Rodrigues, O. M. P. R., \& Capellini, V. L. M. F. (2015). O trabalho do professor, indicadores de Burnout, práticas educativas e comportamento dos alunos: correlação e predição. Revista Brasileira de Educação Especial, 21(3), 363-376. doi: http://dx.doi.org/10.1590/ S1413-65382115000300004 
